\title{
L'information historique des inondations : l'histoire ne donne-t-elle que des leçons ?*
}

\author{
Historical information about floods : does History only give lessons ?
}

\author{
par D. Coeur \\ Université Pierre Mendès France, CRHIPA (Grenoble)
}

M. Lang

Cemagref, Unité de recherche Hydrologie-Hydraulique (Lyon)

Harmonisation among land use planning and natural hazard is now to be improved by the use of historical information. Collaboration between historical and geophysical sciences is intended to give methodological advances, by ensuring the quality of data collection and the technical data processing. This paper presents hydrological and hydraulic requirement for historical information, then the interest of a history of natural hazard, and finally two examples of historical flood studies on Ardèche and Isère rivers.

\section{I INTRODUCTION}

En matière de phénomènes naturels récurrents, d'inondations en particulier, on admet généralement que la connaissance du passé peut participer à la mise en œuvre d'une meilleure prévention du risque. La force de conviction et de vérité délivrée par la mémoire collective, au moment des crises par exemple, montre à quel point la légitimité de l'information historique paraît évidente. Elle est souvent d'ailleurs utilisée pour stigmatiser les responsabilités face à un événement sensé connu ou, à l'inverse, pour rejeter sur le destin la survenue d'un phénomène "inconnu de mémoire d'homme". D'une manière ou d'une autre, le passé vient ainsi naturellement, par l'esprit, à la rencontre du présent et participer un temps aux débats. La récente montée des enjeux autour des risques naturels n'a fait que renforcer l'intérêt pour l'histoire en officialisant en quelque sorte le recours à l'information historique au sein des procédures d'enquête. D'un point de vue judiciaire et en l'absence de toute autre donnée, le juge peut être amené, de son côté, à la retenir pour définir le caractère prévisible d'un événement naturel catastrophique. Il ne reste donc au professionnel de la mémoire qu'à s'exécuter.

Or, derrière cette façade d'évidences se dresse en réalité pour l'historien un décor hybride et disparate. Des chemins

* Extrait du colloque SHF "Gestion des risques liés aux inondations rapides et lentes". balisés, des maisons bien établies, mais aussi des amoncellements d'objets divers, des terrains en friche, entre tout cela beaucoup de vides, et surtout, à l'heure actuelle, aucun plan d'accès, aucune carte permettant d'avoir une vue d'ensemble. On se déplace en tâtonnant ou, au mieux, on utilise des véhicules d'emprunt. Bref, au moment où une discipline aborde ce qui apparaît comme un nouveau champ d'étude, son premier devoir est bien d'abord d'établir une cartographie des lieux. D'un point de vue pratique en effet, et au-delà de la métaphore, tout reste à faire pour transformer en un véritable outil de gestion ce qui relève aujourd'hui davantage de l'intuition que d'une véritable construction scientifique.

La première exigence pour l'historien est donc de se mettre à l'écoute des sciences de la terre, cerner leurs attentes pour établir avec elles les modalités d'une bonne collecte et d'une utilisation maîtrisée de l'information historique sur les inondations. Ce travail est rendu très délicat par le fait que le chercheur, ici peut-être plus qu'ailleurs, se trouve partagé entre l'homme de science et l'expert. De l'un à l'autre le rapport à l'information est bien différent. Cette mobilisation commune ne peut qu'encourager l'historien à s'interroger de son côté sur la réalité et l'intérêt historique du concept de risque naturel tel qu'il émerge aujourd'hui, notamment dans son rapport avec l'aménagement du territoire. Nous resterons volontairement succinct sur ces aspects théoriques généraux. Nous reviendrons en deuxième partie sur le programme de recherche Historisque, exemple concret d'une association ingénieurs-historiens en vue d'une meilleure connaissance des crues extrêmes. 


\section{II — L'INONDATION, LA PRÉVENTION, L'INGÉNIEUR ET L'HISTORIEN}

\subsection{Mémoire et temps de l'inondation}

Que demande à l'histoire l'ingénieur et/ou le scientifique en charge de la connaissance et de la prévention des inondations ? Raconter une histoire ? Non, pas même. Ou alors simplement légitimer son action son projet ou ses analyses : "Regardez ! Lors de l'inondation de 1890 l'eau est venue jusqu'à l'église de ce village, le courant a détruit ces murs de soutènement". On évoque aussi l'effroi des populations, les actes de bravoure... Ici l'ingénieur utilise plus en fait l'imaginaire de son auditoire ou de ses lecteurs pour énoncer sa propre vision des choses, qu'il n'intègre véritablement l'information historique dans ses savoirs et son métier. Car, d'un point de vue plus général, se remémorer l'événement extrême passé, c'est bien d'abord pour la collectivité tenter de mettre en perspective une actualité souvent dramatique. Par le discours s'opère en effet une mise en cohérence entre une réalité extraordinaire, encore incompréhensible, avec une autre toute aussi hors du commun mais déjà intégrée dans les savoirs collectifs et seulement enfouie dans les limbes de la mémoire. La violence de l'événement récent, les excès de destruction, le chaos ont permis de jeter un pont entre le passé et le présent. L'effet cathartique de cette mise en correspondance donne sens à la catastrophe pour mieux lui survivre. Ce registre de référence au passé n'est pas celui des sciences en charge habituellement de définir le risque inondation.

Hydrologues et hydrauliciens utilisent l'information historique en référence d'abord à une définition précise du temps. Il $\mathrm{y}$ a le temps de réalisation du phénomène en lui-même nous n'insisterons pas - mais aussi le temps de la récurrence, celui dans lequel s'inscrivent les événements répétitifs. La répétition d'un événement, dans un espace naturel donné, et sur une période de temps également donnée, constitue en effet, pour l'hydrologue, une série typologique caractéristique du phénomène en question (analyse fréquentielle). L'identité de la famille d'événements ainsi générée est assurée entre autres par l'invariant spatial. On peut, sans doute, et a contrario, discuter de la grande variabilité et de l'évolution des conditions de leur déclenchement (aspects climatiques, géomorphologiques, etc.), points que devra justement éclairer l'historien. Mais une chose est sûre, la définition du second élément, à savoir la donnée temporelle, échappe ici au cadre scientifique habituel d'observation. Ne disposant pas de chroniques de débits historiquement suffisamment longues, l'hydrologue a résolu scientifiquement le problème en extrapolant le comportement d'une série réelle mais restreinte d'observations - quelques décennies - aux phénomènes exceptionnels. Il arrive de cette façon, grâce à la modélisation, le raisonnement et le calcul des probabilités à évaluer le niveau possible des crues extrêmes.

L'information que le scientifique qualifie alors d'historique est bien souvent une appellation générique pour désigner en général celle qui ne provient pas directement hic et nunc de ses instruments de mesure ou des observations effectuées dans des conditions similaires quelques mois ou quelques années auparavant. Elle se situe au-delà de ce laps de temps et de moyens. Il procède alors, dans une première phase, à une quête de données, comme le fait d'ailleurs l'historien. L'évolution des modes de production des données sont un des signes de leur historicité. Mais pour le scientifique, l'in- formation historique ne doit pas avoir d'histoire, ou si elle en a une, sa connaissance n'a paradoxalement d'intérêt que si elle permet de la gommer. La première partie de son travail consistera alors à la mise au point de procédés de transcription entre un cadre informationnel passé générateur d'une famille de données et celui dans lequel il entend travailler désormais. Ce processus de normalisation de l'information est au cœur de la démarche scientifique.

C'est à ce niveau que peut utilement intervenir l'historien, en ouvrant la fenêtre chronologique des séries d'informations disponibles. A partir de la seconde moitié du XVIIIe siècle et plus encore à compter des premières décennies du siècle suivant, l'aménagement, la gestion et la surveillance des cours d'eau ont fait l'objet en France d'une mobilisation spécifique de l'administration des Ponts et Chaussées. On peut compter ainsi aujourd'hui sur des séries d'archives relativement continues dans le temps et homogènes dans leur nature. L'aménagement de chaque cours d'eau peut être suivi de manière assez précise depuis le début du XIXe siècle. Les documents rendent compte aussi des progrès déterminants intervenus, à partir de cette époque, en matière de définition scientifique et technique du territoire (cartes, plans, nivellements, profils, jaugeages, etc.). Analysés de manière systématique, et au terme d'une analyse critique interdisciplinaire, ils délivrent des séries de données potentiellement utilisables par l'ingénieur (cf. variations du profil en long, aménagements successifs du lit majeur, relevés hydrométriques, etc.). Ce contexte documentaire nous a d'ailleurs amené à privilégier la période 1800-2000, l'hypothèse méthodologique de départ étant bien ici de privilégier la mise en cohérence des données techniques "produites" à partir des témoignages "historiques" avec celles actuellement utilisées par les scientifiques. Nous verrons plus bas quelles conséquences pratiques un tel objectif pose en matière de maîtrise quantitative et qualitative de l'information (cf. système d'information).

\subsection{Le scientifique et le devoir de mémoire face au risque inondation}

L'hydraulicien est homme de science mais aussi, à certaines heures, expert. Il assure en quelque sorte dans l'exercice de sa profession, et de manière explicite, une mission de médiation entre le monde de la connaissance des milieux naturels et le reste de la collectivité. Cette rencontre est particulièrement évidente et nécessaire en matière de prévention des risques naturels. Un jeu s'établit entre deux espaces de connaissance et d'appréhension des phénomènes. C'est là, dans le processus - bien décrit par les sociologues - de définition et de gestion sociales du risque que l'ingénieur-expert est aussi confronté à l'histoire. Nous évoquions plus haut la fonction essentielle de la mémoire collective. Elle peut alimenter les polémiques, entraver des négociations. Mise en perspective, elle peut aussi servir à engager le débat, rappeler la permanence des dangers, la nécessité d'agir et les obligations de chacun, etc. Encore faut-il que ce passé soit connu et/ou reconnu des habitants. Si une mémoire subsiste, on veillera donc non seulement à la compléter mais encore à l'associer, sous une forme ou une autre, à l'étude en cours. L'objectif est bien de faire des habitants les premiers propriétaires de l'information historique. On pourra ensuite plus facilement lui associer des informations techniques à caractère préventif. Ces modalités de traitement de l'information ne relèvent pas toutes des compétences de l'ingénieur. Ici le devoir collectif de mémoire cherche encore des formes et 
modes d'inscription adaptés à son objet et aux populations concernées. Sans doute l'outil Internet pourrait-il assurer en partie cette interface. Quant à l'historien, il semble associé presque malgré lui à cette instrumentation du passé.

\subsection{Risques naturels et aménagement :}

\section{les nouveaux territoires de l'historien}

Face à ces interrogations multiples de la collectivité en direction des risques naturels et dont les sciences de la terre se font l'écho, quelle est la position de l'historien ? En d'autres termes, les questions qui se posent aujourd'hui sur le plan scientifique, technique, politique, social ou culturel dans ce champ, et dont certaines sciences sociales ont déjà rendu compte - sociologie, sciences politiques, droit -, ontelles une réalité historique plus large ? Nous n'essaierons pas ici de répondre directement à cette question. Ce n'est pas le lieu. Il nous semble important par contre de souligner les lacunes actuelles de l'historiographie dans ce domaine, lacunes qui rendent plus délicat encore le travail entre l'ingénieur et l'historien.

En fait, comme le souligne Christian Desplat [1], il n'existe pas encore de véritable histoire des risques naturels. Ce serait sans doute devoir reconnaître à la nature une place par trop importante dans la production des territoires, alors que la discipline historique a fait fi depuis longtemps de tout déterminisme. La géographie elle-même a renoncé à cette approche. Mais pour l'historien adepte du temps long cher à l'Ecole des Annales, c'est aussi et surtout la place (re)donnée indirectement à l'événement qui déroute ici son analyse. Sans compter qu'il devra aussi intégrer l'histoire des sciences et techniques qui ont participé à la maîtrise des phénomènes naturels. Et quand on sait la place réservée à cette discipline dans l'enseignement de l'histoire en France, on comprendra là encore les difficultés actuelles.

Dans la mouvance des grands courants historiographiques contemporains, les chercheurs ont avant tout abordé la question des phénomènes naturels violents par le registre culturel. Décrire et comprendre l'évolution des perceptions et représentations du milieu naturel est essentiel mais n'aborde qu'une facette de la question. "L'invention des paysages" de montagne par exemple est un cas d'école comme le soulignent dernièrement encore les travaux de Numa Broc [2] ou de Serge Briffaud [3]. Le risque dans son acception moderne est bien à resituer dans l'espace de rencontre entre nature et collectivité humaine, cette dernière étant plus ou moins consciente et capable de mobiliser des moyens pour se préserver de la destruction. Les travaux pionniers, mais déjà anciens, d'Emmanuel Le Roy Ladurie [4] sur le climat, restent isolés. L'auteur soulignait l'intérêt d'une telle analyse pour comprendre certains cycles de crises socio-économiques, mais reconnaissait aussi déjà la nécessité d'un travail interdisciplinaire pour avancer dans une connaissance scientifique plus poussée du climat et de son évolution. Par contre il n'évoque pas la possibilité de s'engager vers la mise en œuvre d'une véritable histoire du milieu naturel. Histoire plus générale de l'environnement qui soit délivrée du regard esthétique qui réduit la nature au seul paysage. (...) Ce n'est pas parce que l'homme a sa manière à lui d'être historique que la nature ne peut avoir sa manière à elle de l'être [5]. La nature peut avoir une "histoire" en marge de celle de l'homme. La connaissance des phénomènes naturels récurrents en est un exemple.

Dans cette veine, on peut s'interroger alors sur la pertinence d'une histoire de la maîtrise de l'espace naturel par la collectivité des hommes. L'émergence actuelle du concept de risque n'est pas fortuite. Elle s'inscrit dans une histoire beaucoup plus longue de l'aménagement de la nature. Or c'est bien connu, on fait l'histoire des vainqueurs, rarement celle des vaincus. Pendant des siècles, l'homme a dû se défendre et lutter contre les éléments naturels. Si aujourd'hui une histoire du risque naturel devient possible, c'est bien parce que notre regard sur la nature a effectivement changé au cours des dernières décennies. Celle-ci commence à être respectée pour elle-même, préservée et a même acquis le statut de patrimoine... Bref, on commence à lui prêter une identité à part entière, identité que l'on a désormais conscience d'avoir depuis très longtemps négligée, sinon bafouée. L'historiographie en est une nouvelle fois témoin. Les premiers travaux à caractère historique sur les risques naturels qui empruntent ce nouveau regard sont l'œuvre de géographes [6] et non d'historiens. Des pans entiers de l'histoire politique, administrative, scientifique et technique relative à l'aménagement des espaces naturels sont en friche ou à revisiter. Cette relecture de l'histoire ne pourra qu'enrichir l'appareil critique de l'historien et de l'ingénieur dans leur analyse commune des phénomènes naturels passés.

\section{III $\square$ LE PROJET HISTORISQUE : VERS UNE MAÎTRISE DE L'INFORMATION HISTORIQUE DES INONDATIONS}

Ce projet a été engagé sur la rivière du Guiers en 1995 et se poursuit actuellement sur l'Ardèche et l'Isère. Il a obtenu le soutien du Pôle Grenoblois pour l'Etude et la Prévention des Risques Naturels depuis cette date, du Contrat de Plan Etat Région Rhône-Alpes en 1998-1999 et du Cemagref en 1998-1999. Il associe principalement l'unité de recherche hydrologie-hydraulique du Cemagref (Lyon) et le laboratoire CRHIPA de l'université Pierre Mendès France.

\subsection{Une enquête historique d'abord au service de la science hydraulique}

L'objectif principal est de tester l'intérêt de l'information historique dans l'analyse des crues extrêmes. L'hypothèse part d'un constat relativement simple. L'analyse des chroniques de débits qui fonde aujourd'hui l'étude statistique des périodes de retour des phénomènes exceptionnels, repose sur des séries de relevés trop limitées dans le temps, quelques décennies seulement. Le but du projet est d'élargir l'échantillon de plusieurs décennies et au mieux de l'étendre aux deux derniers siècles. Les résultats issus de ces grandes séries historiques serviront de référence pour tester le comportement de méthodes fréquentielles extrapolant les crues extrêmes à l'aide de l'information pluviométrique (Gradex, Agregee). Nous avons donc affaire ici à une mobilisation restreinte de l'histoire et $a$ priori à une collecte relativement simple de données sous la forme d'une chronologie d'événements (niveau de crues) au droit de sites d'observation.

En fait, si les scientifiques maîtrisent à peu près les paramètres entrant aujourd'hui dans une analyse de ce genre (définition topographique du cours d'eau, jaugeages, etc.), la remontée progressive dans un passé de plus en plus éloigné des techniques contemporaines d'investigation et de mesure demande un suivi précis de toute une série de paramètres. Faute de quoi, les calculs et analyses ultérieurs risqueraient d'être entièrement faussés. 


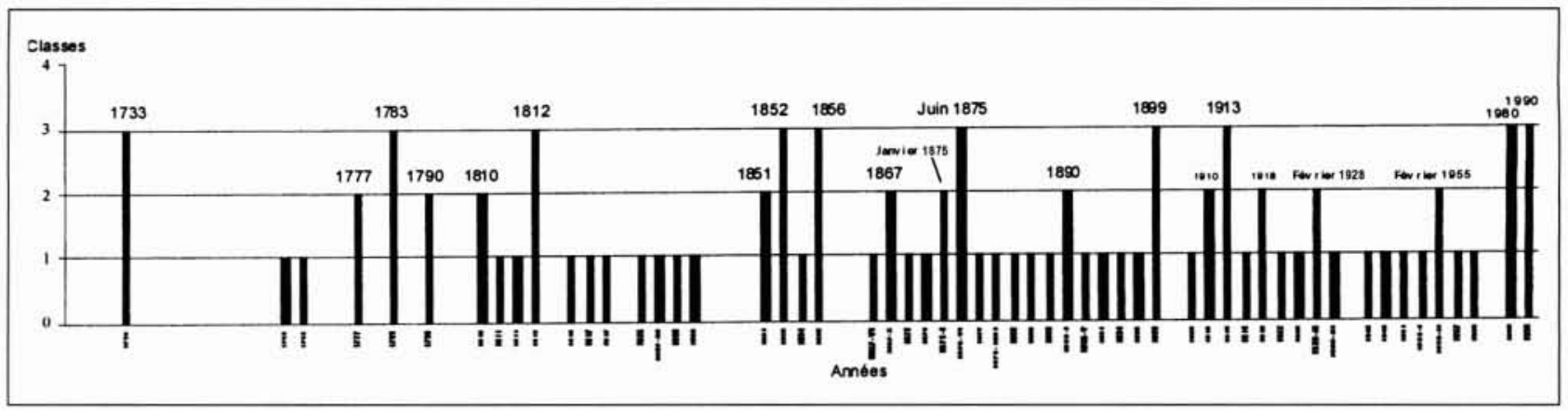

1. Essai de classement des crues - Exemple de la rivière du Guiers (XVIIIe-XXe s.).

Cette exigence critique a débouché sur la mise au point d'une grille de lecture des informations historiques sur les crues. Elle permet de mettre en rapport les objectifs méthodologiques avec les types de données à rechercher. La lecture de ce tableau peut s'opérer dans un sens comme dans l'autre (Tableau 1). Les informations sont regroupées en trois grands ensembles. Le premier renseigne sur les services producteurs des grandes catégories de données (topographie, hydrométrie, etc.). Le second est consacré aux méthodes et matériels de mesure mis au point et utilisés dans ces différents domaines. Le troisième est de loin le plus important. Il classe l'ensemble des événements du bassin hydrographique étudié, la notion d'événement étant entendue ici au sens large du terme. Ce sont bien sûr toutes les informations relatives directement aux crues et inondations (cotes, débits, champs d'inondation) mais également les aménagements importants intervenus dans le lit (digues, barrages) ou encore dans le bassin (urbanisation, déforestation). Toute opération de mesure ou de relevé (pluviométrie, lignes d'eau, etc.) est aussi considérée comme un événement.

Trois ensembles de données sont particulièrement remarquables compte tenu de l'espace chronologique considéré et des méthodes employées. La mise au point d'une chronologie qualitative des crues de la rivière permet d'abord de repérer dans le temps les événements extrêmes. Ils feront par la suite l'objet d'une analyse spécifique dans le cadre de l'étude (Figure 1). Chaque classe identifie une famille d'événements établie selon un certain nombre de critères. Le graphique est accompagné d'un tableau analytique et chronologique donnant une description détaillée de chaque phénomène qui précise la nature de la documentation utilisée et renvoie si nécessaire directement à des documents majeurs qui peuvent alors être reproduits en annexe (plans, graphiques, photos, etc.). La définition topographique du cours d'eau utilise tout particulièrement ce type de témoignage. Collationnés et référencés avec précision, ils permettent de disposer au terme de l'enquête de la collection complète des plans, profils se rapportant à la rivière et ce sur les deux derniers siècles voire plus sur les grands cours d'eau (deux siècles et demi par exemple pour l'Isère). Les relevés hydrométriques journaliers sur station constituent le troisième principal corpus de données. Ces observations systématiques ont été mises en place en France sur les grands cours d'eau au cours de la seconde moitié du XIXe siècle. Nous disposons par exemple pour l'Isère de relevés systématiques, ou presque, entre 1867 et nos jours. Toutes ces cotes ont été saisies sur support informatique pour faire ensuite l'objet d'un traitement informatique spécifique lors des modélisations hydrauliques.

\subsection{Maîtriser l'information}

Au fur et à mesure de l'avancement de l'enquête, la maîtrise quantitative et qualitative de l'information est devenue un des maillons essentiels du projet. Elle s'est concrétisée pour l'instant par deux éléments distincts. La grille de lecture présentée ci-dessus d'une part, et la mise au point de ce que nous avons appelé l'état général des sources (EGS), d'autre part. L'EGS dresse le paysage documentaire général dans lequel se déploie l'enquête (souci d'exhaustivité et de cohérence). La grande dispersion des sources d'information tant actuelles que passées en matière hydraulique nécessitait la mise au point d'un tel tableau de référence, fondement d'une approche critique des données historiques. Nous avons veillé tout particulièrement à la lisibilité de ce document et à ses possibilités de mise à jour. Deux hypothèses ont orienté sa réalisation. Premièrement le souci de continuité entre les données actuellement disponibles couvrant en général les deux ou trois dernières décennies et celles relatives aux périodes immédiatement antérieures. Considérer, deuxièmement, le tournant de la fin du XVIII siècle comme la borne chronologique haute de l'enquête, non pas que nous rejetions toute information antérieure car l'analyse statistique des crues extrêmes intègre avec grand intérêt les données plus anciennes. Comme nous le rappelions plus haut, cette période marque en fait l'apparition et le développement d'une première documentation technique de qualité qui seule présente les conditions d'information suffisantes pour mener à bien les analyses hydrauliques ultérieures.

Cet état général des sources, en voie d'achèvement, est consigné dans une base de données où sont stockées les références de chaque source d'information : numéro de référence, auteurs, année, titre, mots-clés, localisation archive, fiche résumé. La consultation sélective des différentes informations collectées s'effectue par le biais de requêtes basées sur les mots-clés de la grille de lecture. Elle permettra de reconstituer l'historique des producteurs de données en matière d'inondation, et éventuellement de mettre en évidence des sources d'information qui n'auraient pas encore été explorées. L'historique de la mesure sera exploité pour positionner dans l'espace les données qui concernent la topographie et pour estimer les incertitudes liées aux méthodes propres à chaque époque, tant en hydrométrie qu'en matière de pluviométrie. Enfin, l'historique des événements sera l'occasion d'un travail de reconstitution du débit des principales crues historiques.

L'information historique sera alors utilisée, soit comme preuve de catastrophes du passé (cf. culture du risque, exper- 


\begin{tabular}{|c|c|}
\hline THEME & OBJECTIF \\
\hline \multicolumn{2}{|l|}{1 - Historique des services en charge de : } \\
\hline la topographie & \multirow{4}{*}{ Cibler les sources éventuelles d'informations } \\
\hline l'hydrométrie et de l'annonce des crues & \\
\hline la météorologie & \\
\hline $\begin{array}{l}\text { la gestion de l'aménagement du territoire (forêt, travaux } \\
\text { publics, énergie hydroélectrique) }\end{array}$ & \\
\hline \multicolumn{2}{|l|}{2 - Historique des méthodes et matériels de mesure : } \\
\hline Topographie & Situer spatialement les données historiques \\
\hline Hydrométrie & \multirow{4}{*}{$\begin{array}{l}\text { Estimer les incertitudes liées } \\
\text { aux pratiques de l'époque }\end{array}$} \\
\hline . Enregistrement des hauteurs d'eau & \\
\hline . Estimation des débits (jaugeages) & \\
\hline . Conversion hauteur-débit (courbe de tarage) & \\
\hline \multirow{2}{*}{\multicolumn{2}{|c|}{$\begin{array}{l}\text { Pluviométrie } \\
\text {. Enregistrement des hauteurs d'eau }\end{array}$}} \\
\hline & \\
\hline \multicolumn{2}{|l|}{3 - Historique des événements du bassin : } \\
\hline \multicolumn{2}{|l|}{ Les aménagements (construction, destruction) } \\
\hline . Barrages, ponts, digues & Modélisation hydraulique des crues \\
\hline . Forêts, urbanisation & Modifications hydrologiques \\
\hline Les opérations de topographie & \multirow{2}{*}{ Evolution morphologique du lit } \\
\hline . Vue en plan, profils en travers, profils en long & \\
\hline \multicolumn{2}{|l|}{ L'hydrométrie } \\
\hline . Dossiers des stations hydrométriques et limnigraphes & Référencement altimétrique des hauteurs de crue \\
\hline . Jaugeages, barême des débits, courbes de tarage & \multirow{4}{*}{ Modélisation hydraulique } \\
\hline Crues & \\
\hline $\begin{array}{l}\text {. Relevés de cotes et de débits (hauteur maximale, } \\
\text { hauteur journalière, débits maxima, débits journaliers) }\end{array}$ & \\
\hline . Limites de champ d'inondation & \\
\hline Etiages & \multirow{3}{*}{ Evolution morphologique du lit } \\
\hline . Relevés de cotes et de débits & \\
\hline . Levés de la ligne d'eau & \\
\hline Réglementation & $\begin{array}{l}\text { Evolution de la prise en compte du risque } \\
\text { d'inondation }\end{array}$ \\
\hline La pluviométrie & \multirow{3}{*}{$\begin{array}{l}\text { Méthodes d'extrapolation des distribution de crue } \\
\text { (Gradex, Agregee) }\end{array}$} \\
\hline . Dossiers des stations pluviométriques & \\
\hline $\begin{array}{l}\text {. Relevés de précipitations (pluies mensuelles, } \\
\text { pluies journalières) }\end{array}$ & \\
\hline Climatologie (sécheresse ; périodes froides) & Stationnarité \\
\hline
\end{tabular}

Tableau 1 - Grille de lecture des informations historiques sur les crues.

tise judiciaire post-catastrophe, événement de référence dans le zonage du risque), soit comme donnée hydrologique incorporée dans le calcul des probabilités d'occurrence. Elle devient alors l'un des éléments d'aménagement et de gestion du territoire. A ce stade, la maîtrise qualitative de l'information historique est essentielle et il importe de prendre en considération les éléments pouvant perturber cette connaissance de l'aléa : l'exhaustivité et la fiabilité des sources historiques, la stationnarité de la population des crues à l'échelle de deux siècles, et les incertitudes sur la reconstitution du débit des crues.

\section{IV $\square$ CONCLUSION}

Au total, si la remémoration du passé apparaît comme une manifestation naturelle lors d'une inondation catastrophique, l'information véhiculée à cette occasion par la mémoire collective est loin toutefois de revêtir tous les caractères de la vérité historique. La connaissance "scientifique" des phénomènes naturels passés est envisageable seulement à partir du moment où les questions posées par les sciences de la terre peuvent être inscrites dans la critique historique. Le travail interdisciplinaire consistera donc d'abord à valider toute une 
série de pratiques et d'informations. L'outil informatique ouvre de ce point de vue des perspectives nouvelles. Pour autant, le rapprochement entre disciplines ne réside pas uniquement dans la réalisation ponctuelle d'un bel outil. A l'instar de ce qui se fait déjà depuis plusieurs années dans le champ des paléo-événements, le travail transdisciplinaire qui s'organise en matière de connaissance historique des inondations a besoin de s'inscrire dans le temps. A ce titre seulement il pourra prétendre au statut de nouvelle expertise des territoires.

\section{BIBLIOGRAPHIE}

[1] Desplat C. [1996] - Pour une histoire des risques naturels dans les Pyrénées occidentales françaises sous l'Ancien Régime, Les Catastrophes naturelles dans l'Europe médiévale et moderne, Actes du colloque Flaran XV de septembre 1993, Toulouse, P.U.M., 115-163.

[2] Broc N. [1991] - Les montagnes au siècle des Lumières. Perception et représentation, Comité des Travaux Historiques et Scientifiques Mémoires de la section de géographie physique et humaine, $n^{\circ} 4$, Paris, Edition du C.T.H.S.

[3] Briffaud S. [1994] - Vision et représentation du paysage montagnard (Pyrénées Centrales milieu XVIIIe-milieu XIXe s.). Essai sur l'histoire culturelle des relations des sociétés à leur environnement, Toulouse, A.G.M./Archives des Hautes-Pyrénées/C.I.M.A.-C.N.R.S.

[4] Le Roy Ladurie E. [1974] - Le climat : histoire de la pluie et du beau temps, dans Faire de l'Histoire, III, Nouveaux objets, sous la direction de Jacques Le Goff et Pierre Nora, Paris, Gallimard (Folio-Histoire), 1974, 11-46.

[5] Veyne P. [1971] - Comment on écrit l'histoire. Essai d'épistémologie, Paris, Le Seuil, p. 21.

[6] Desailly B. [1990] - Crues et inondations en Roussillon. Le risque et l'aménagement fin XVIle-mi XXe siècle siècles, Thèse de géographie, Paris X-Nanterre.
[7] Antoine J.-M. [1992] - La catastrophe oubliée. L'inondation et l'aménagement de la vallée de l'Ariège fin XVIle-XXe siècle. Thèse de géographie, Toulouse Le Mirail.

Travaux rentrant dans le cadre des projets Historisque

[8] Ceur D., Lang M., Naulet R., Burnet R., Strazzeri D. [1998] - Histoire et connaissance des phénomènes naturels extrêmes - Ingénieries EAT - numéro spécial Risques Naturels, 15-26.

[9] Ceur D., Gigon C., Lang M., Naulet R. [1999] - HistorisqueArdèche : utilisation de l'information historique pour une meilleure définition du risque d'inondation - rapport première année, Contrat de Plan Etat-Région Rhône-Alpes, juillet, 55 p.

[10] Cerur D., Naulet R., Lang M., Soulingeas Y. [1999] - HistorisqueIsère : utilisation de l'information historique pour une meilleure définition du risque d'inondation - rapport intermédiaire, Pôle Grenoblois Risques Naturels, mars, 35 p.

[11] Lang M., Cæur D., Lallement C., Naulet R. [1998] - Use of historical information for flood frequency studies : the example of river Guiers - Séminaire annuel FRIEND-AMHY, UNESCO IHP l, Istambul, Turquie, 14-16 oct. 1998.

[12] Lang M., Cæur D., Lallement C., Naulet R. [1998] - Valorisation de l'information historique pour la prédétermination du risque d'inondation : application sur le bassin du Guiers - Ingénieries EAT - n ${ }^{\circ} 16$. décembre, 3-13.

[13] Naulet R. [1999] - Utilisation de l'information historique pour une meilleure prédétermination du risque d'inondation - Document préparatoire à l'examen doctoral. Cemagref Lyon, Univ. J. Fourier Grenoble. INRS-Eau. Québec, 16 août, 57 p.

[14] Naulet R., Lang M. [1999] - Collaboration between historians and hydrologists on the Ardeche river (France) : use of historical information for flood frequency analysis - EGS, XXIX General Assembly, NH3.3 "The use of historical data in natural hazard assessment : Floods. Poster session, The Hague, Netherlands, 19-23 April. 